\title{
The temporal dynamics of detached versus positive reappraisal: An ERP study
}

\author{
Senqing $\mathrm{Qi}^{1}$ • Yangping $\mathrm{Li}^{1,2} \cdot$ Xuemei Tang ${ }^{1} \cdot \mathrm{Qinghong} \mathrm{Zeng}^{3} \cdot$ Liuting Diao $^{4}$ • \\ Xiying $\mathrm{Li}^{1,2} \cdot$ Hong $\mathrm{Li}^{5} \cdot$ Weiping $\mathrm{Hu}^{1,2}$
}

Published online: 23 January 2017

(C) Psychonomic Society, Inc. 2017

\begin{abstract}
Detached reappraisal and positive reappraisal are regarded as two distinct types of cognitive reappraisal strategy, and the former is considered more disengaging than the latter. The conceptual framework of emotion regulation choice posits that strategies involving disengagement operate earlier and more efficiently than engagement strategies. Here, we compare for the first time the temporal dynamics of detached and positive reappraisal during the regulation and re-exposure phases thereof by measuring event-related potentials. During the regulation phase, pictures were viewed or regulated using detached or positive reappraisal. During the re-exposure phase, the same pictures were viewed again. Results showed that during regulation, central-parietal late positive potentials (LPPs) were greatly attenuated under both strategy types, with the regulation effect of detached reappraisal occurring earlier than that of positive reappraisal and resulting in a stronger
\end{abstract}

Electronic supplementary material The online version of this article (doi:10.3758/s13415-016-0494-4) contains supplementary material, which is available to authorized users.

Senqing Qi

qisenqing@126.com

Weiping $\mathrm{Hu}$

weipinghu@163.com

1 MOE Key Laboratory of Modern Teaching Technology, Shaanxi Normal University, Xi' an 710062, China

2 Collaborative Innovation Center of Assessment for Basic Education Quality, Beijing, China

3 Department of Psychology, The Chinese University of Hong Kong, Shatin, N. T. 999077, Hong Kong

4 MOE Key Laboratory of Cognition and Personality, Southwest University, Chongqing 400715, China

5 Psychology \& Social College, Shenzhen University, Shenzhen 518060, China attenuation of LPP amplitudes. Upon re-exposure, detached reappraisal exerted enduring effects on self-reported arousal and the central-parietal LPP, whereas positive reappraisal had an enduring effect only on pleasantness. These findings demonstrate the differential effects of detached and positive reappraisal on valence, arousal, and neural responses, and underline the striking differences in the temporal dynamics of these reappraisal strategies.

Keywords Emotion regulation $\cdot$ Reappraisal $\cdot$ Event-related potential $\cdot$ Late positive potential

Emotion regulation enables people to deal with the affective ups and downs of daily life and can be implemented by different strategies. Cognitive reappraisal is a highly effective regulation strategy; it aims to alter one's emotional response by encouraging one's reinterpretation of the emotional stimulus' meaning (Buhle et al., 2014). Most previous research has focused on comparing cognitive reappraisal with other strategies, while little has sought to assess differences between subtypes of cognitive reappraisal. Such comparative research is of great importance, as cognitive reappraisal is not a homogenous regulation strategy, but instead comprises several specific strategies that differ with respect to the way the individual reinterprets the emotional stimulus (McRae, Ciesielski, \& Gross, 2012; Ochsner et al., 2004). The most commonly studied reappraisal substrategies are detached reappraisal and positive reappraisal (Shiota \& Levenson, 2012), which are frequently used interchangeably (Moser, Krompinger, Dietz, \& Simons, 2009; Paul, Simon, Kniesche, Kathmann, \& Endrass, 2013; Thiruchselvam, Blechert, Sheppes, Rydstrom, \& Gross, 2011). Detached reappraisal involves reinterpreting emotional stimuli in a detached and unemotional way, while positive reappraisal does so in a positive way. 
These reappraisal strategies differ in their effectiveness and involve distinct neural networks. Detached reappraisal is effective at reducing the emotional intensity of negative stimuli, whereas positive reappraisal tends to shift one's emotional experience toward positive affect (Denny \& Ochsner, 2014; Shiota \& Levenson, 2012). Furthermore, detached, but not positive, reappraisal is effective at down-regulating amygdala activity, recruiting a right prefronto-parietal regulation network, as compared to the left frontal control network recruited during positive reappraisal (Dorfel et al., 2014). However, these studies failed to provide the timeframe of these strategies' emotion-regulating effects.

Given the importance of time course in emotion regulation research and theory (Gross, 2014; Hajcak, Dunning, Foti, \& Weinberg, 2014), event-related potentials (ERPs) as a measurement technique with excellent temporal resolution should be used for the comparison of detached and positive reappraisal's temporal dynamics. ERP research has identified the stimulus-preceding negativity (SPN) and late positive potential (LPP) waveforms related to cognitive reappraisal. The SPN is primarily observed during anticipation of emotional stimuli; it reflects anticipatory processes in preparation for dealing with the upcoming stimulus (Brunia, van Boxtel, \& Böcker, 2012; Moser, Hartwig, Moran, Jendrusina, \& Kross, 2014; Seidel et al., 2015). When detached and positive reappraisal are used interchangeably, cognitive reappraisal cues elicit larger frontal SPNs than simple viewing cues, suggesting enhanced preparation for engaging in the reappraisal processes (Moser et al., 2009; Thiruchselvam et al., 2011). However, when positive reappraisal is used alone, positive reappraisal cues do not modulate SPN amplitudes (Moser et al., 2014).

The LPP is widely recognized as a central-parietal ERP that reflects enhanced processing of emotional stimuli deemed more arousing than those of neutral valence (Cuthbert, Schupp, Bradley, Birbaumer, \& Lang, 2000; Hajcak, MacNamara, \& Olvet, 2010; Hajcak et al., 2014). The central-parietal LPP is sensitive to cognitive reappraisal (Hajcak \& Nieuwenhuis, 2006; Hajcak et al., 2014) and can be used to track emotion regulation strategies' temporal dynamics (Paul et al., 2013; Schönfelder, Kanske, Heissler, \& Wessa, 2014; Thiruchselvam et al., 2011). Additionally, an enhanced frontal LPP, indicating cognitive effort or attentional control, is observed during positive reappraisal implementation (Moser et al., 2014; Shafir, Schwartz, Blechert, \& Sheppes, 2015) and the free implementation of detached and positive reappraisal (Moser et al., 2009). It therefore remains unclear whether enhanced frontal LPP is specifically associated with positive reappraisal.

Recently, Willroth and Hilimire (2016) investigated regulation effects of self- and situation-focused reappraisal on electrocortical responses to negative pictures. They found that situation-focused reappraisal (similar to positive reappraisal), but not self-focused reappraisal (similar to detached reappraisal), was associated with a reduction of the centralparietal LPP amplitude. However, this study did not offer the time course of the LPP; therefore, the temporal dynamics of detached and positive reappraisal is still unclear. In addition to understanding the momentary effects of detached and positive reappraisal during implementation, their enduring effects on re-encountered stimuli also merit examination, as emotionally stimuli often recur in daily life. Therefore, this study used ERP to compare the temporal dynamics of detached and positive reappraisal during the regulation and re-exposure phases. In the regulation phase, pictures were viewed or regulated using detached or positive reappraisal. In the re-exposure phase, the same pictures were viewed passively.

In the regulation phase, based on previous research (Shiota \& Levenson, 2012; Willroth \& Hilimire, 2016), we hypothesized that detached reappraisal would effectively reduce selfreported arousal in response to negative pictures, whereas positive reappraisal would effectively increase pleasantness. In detached reappraisal, participants could prepare to adopt a detached attitude before the picture's presentation. In contrast, in positive reappraisal, such preparation is impossible as positive reinterpretation of emotional stimuli can occur only after their initial interpretation. We thus predicted that detached reappraisal cues, as compared with positive reappraisal cues, would elicit enhanced SPN that index preparatory activity. Reappraisal is generally considered an engagement regulation strategy. Nevertheless, detached reappraisal is considered more disengaging than positive reappraisal, as it involves disengaging from the stimulus' emotional implications (McRae et al., 2012; Sheppes et al., 2014). According to the conceptual framework of emotion regulation choice proposed by Sheppes et al. (2014), disengagement strategies operate earlier and more efficiently than engagement strategies. Therefore, detached reappraisal ought to result in earlier and greater attenuation of central-parietal LPPs than positive reappraisal. Furthermore, as additional cognitive effort is required for positively reappraising negative pictures (Dorfel et al., 2014), we expected that positive, but not detached, reappraisal would elicit larger frontal LPPs, indicative of cognitive effort.

Both reappraisal strategies involve altering the appraisal of emotional stimuli; therefore, the modified meaning may be reactivated on re-presentation of these stimuli (Macnamara, Ochsner, \& Hajcak, 2011; Thiruchselvam et al., 2011). Detached reappraisal tends to push the meaning of emotional stimuli in a neutral direction; therefore, a more neutral interpretation of negative pictures may be reactivated upon re-exposure. During the re-exposure phase, we thus predicted that negative stimuli with a detached reappraisal history would be rated as less arousing and elicit smaller central-parietal LPPs than those with a view history. Positive reappraisal shifts one's emotional experience toward positive affect; therefore, a more pleasant interpretation of negative pictures may be reactivated. Consequently, negative stimuli with a positive reappraisal 
history would be rated as more pleasant than those with a view history.

\section{Method}

\section{Participants}

Twenty-seven undergraduate students voluntarily participated in the ERP experiment, with 40 yuan as payment. Two participants were excluded because of excessive artifacts $(>50 \%$ of rejected trials). Therefore, the final sample consisted of 25 participants (15 females; mean age $=20.48$ years, $S D=$ 1.23). All participants had normal or corrected-to-normal vision and reported no history of psychiatric diseases.

\section{Stimuli}

One hundred and twelve pictures (84 negative, 28 neutral), used by Thiruchselvam et al. (2011), were chosen from the International Affective Picture System (IAPS; Lang et al., 2008). As culture-related differences in affective ratings of IAPS pictures have been reported in Chinese subjects (Huang \& Luo, 2004), these pictures were assessed on a 1-9 scale in terms of valence (1 [unpleasant] to 9 [pleasant]) and arousal (1 [low] to 9 [high]) by a separate group of 18 participants (10 females; mean age $=19.44$ years, $S D=0.78)$ to ensure the rationality of picture classification. The two categories differed significantly from each other on valence rating (neutral pictures: $M=5.28, S D=0.44$; negative pictures: $M=$ 2.91, $S D=0.55), t(17)=13.19, p<.001$, and arousal rating (neutral pictures: $M=4.42, S D=1.06$; negative pictures: $M=$ $6.17, S D=1.01), t(17)=-8.37, p<.001$. Then, 84 negative pictures were divided into three sets of 28 pictures (Sets A, B, and $C),{ }^{1}$ and these three sets did not differ in valence or arousal ratings $(p s>.40)$.

\section{Procedure}

After providing informed consent, participants received a detailed explanation of how to implement detached and positive reappraisal. They then practiced each reappraisal strategy with 10 negative pictures (which were not presented in the formal experiment) with

\footnotetext{
${ }^{1}$ The ID numbers of the IAPS pictures used were the following: Set A (1050, 1300, 1930, 2661, 2700, 3064, 3100, 3101, 3140, 3181, 3220, 3230, 3301, $3350,3550,6415,6830,7359,7380,8230,9040,9042,9181,9433,9570$, 9571, 9622, 9810); Set B (1111, 1201, 2095, 2141, 2399, 2710, 2750, 2800, 3030, 3051, 3053, 3060, 3168, 6211, 6313, 6315, 6350, 6510, 6550, 6555, $6821,9006,9252,9253,9420,9421,9635,9921)$; Set C (2120, 2130, 2375, 2683, 2691, 2810, 2900, 2981, 3000, 3010, 3015, 3120, 3130, 3160, 3400, $3530,6212,6230,6243,6570,6831,9050,9250,9265,9300,9301,9405$, 9410); Neutral (2190, 2221, 2235, 2280, 2320, 2383, 2393, 2394, 2440, 2480, 2495, 2516, 2579, 2580, 2593, 2749, 2840, 2850, 2870, 7025, 7090, 7175, 7211, 7217, 7493, 7496, 7550, 9210).
}

guidance and feedback from the experimenter. In the practice experiment, participants were asked to narrate aloud their reinterpretation of pictures according to preceding cues. The formal experiment did not begin until participants were able to implement each reappraisal strategy successfully. The formal experiment comprised the regulation task and the re-exposure task.

\section{Regulation task}

Participants were cued to respond to pictures either by simply viewing them without employing any strategy ("simple viewing" hereafter) or by implementing detached or positive reappraisal. Each trial started with the presentation of a whitegray fixation cross for $0.5 \mathrm{~s}$. An instruction cue (Chinese translation of "simple viewing," "detached reappraisal," or "positive reappraisal") was then presented for $2 \mathrm{~s}$, signaling to the participants how to respond to the upcoming picture. A blank screen was then presented for $500 \mathrm{~ms}$, followed by a fixation cross for a further $500 \mathrm{~ms}$. IAPS pictures were then displayed on the full screen of a 24-inch monitor for $5 \mathrm{~s}$. Subsequently, participants were asked to rate these pictures on the dimensions of valence (1 [unpleasant $]$ to 9 [pleasant $])$ and arousal (1 $[l o w]$ to $9[$ high]). The inter-trial interval was $2 \mathrm{~s}$.

During simple viewing, participants were asked to view neutral or negative pictures and let any emotions they felt come and go naturally. During positive reappraisal, participants were asked to reinterpret the cause, outcome, or significance of the pictured events in a positive way so that they would feel less negative emotions. For example, on seeing a picture of a woman and man wrestling, one might imagine that the woman is in selfdefense simulation training, or that the woman finally subdues the bad man. During detached reappraisal, participants were asked to think about the pictured events from a detached and unemotional perspective so that they would feel less negative emotions. Returning to the aforementioned example of a woman and man wrestling, now through the lens of detached reappraisal, one might judge the pictured event as having nothing to do with one's own affairs, or as being far away, or perhaps even imagine that it is not real.

We conducted four types of trials: simple viewing of neutral pictures ("neutral-view"), simple viewing of negative pictures ("negative-view"), positive reappraisal of negative pictures ("positive reappraisal"), and detached reappraisal of negative pictures ("detached reappraisal"). The three sets of negative pictures (Sets A, B, and C) were randomly assigned to the negative-view, positive reappraisal, and detached reappraisal trials. Based on a prior study (Thiruchselvam et al., 2011), the block manipulation of reappraisal strategies was used to avoid their mixture. A total of 112 trials were equally divided into four blocks. Each block included seven neutralview, seven negative-view, and 14 positive-reappraisal or 14 detached-reappraisal trials. The sequence of the 28 trials 
within each block was separately randomized for each subject, and the order of the blocks was counterbalanced.

After completion of the regulation task, participants were asked to assess (a) how successfully they complied with the instructions to implement each reappraisal strategy (1: not at all successful, 10: very successful) and (b) how much effort they expended to implement each reappraisal strategy (1: expended no effort at all, 10: expended great effort).

\section{Re-exposure task}

After an interval of approximately $30 \mathrm{~min}$, during which they completed individual differences measures ${ }^{2}$ and had a rest, participants were asked to simply view the same pictures that were presented during the regulation task. Participants had not been told that they would be re-exposed to these pictures. The re-exposure task consisted of 112 trials, which were also divided into four blocks. Each trial began with the presentation of a fixation cross for $0.5-1 \mathrm{~s}$, followed by a full-screen picture for $3 \mathrm{~s}$. Participants were then asked to rate the pictures on the dimensions of valence and arousal.

\section{Electroencephalographic recording and processing}

Raw electroencephalographic (EEG) data were recorded at 64 scalp sites using $\mathrm{Ag} / \mathrm{AgCl}$ electrodes, and referenced to the left mastoid, with a ground electrode on the medial frontal aspect. The vertical electrooculogram (EOG) was recorded supra- and infra-orbitally at the left eye; the horizontal EOG was recorded from the left versus the right orbital rim. The EEG and EOG were amplified by a SynAmps2 amplifier (Neuroscan, Herndon, VA, USA) and digitized at $500 \mathrm{~Hz} /$ channel with a band-pass filter of DC to $100 \mathrm{~Hz}$. All interelectrode impedances were maintained below $5 \mathrm{k} \Omega$.

Off-line analyses were performed in MATLAB using the EEGLAB toolbox (Delorme \& Makeig, 2004) and ERPLAB toolbox (Lopez-Calderon \& Luck, 2014). EEG data were down-sampled to $250 \mathrm{~Hz}$, rereferenced to the average of the left and right mastoids, and filtered using Butterworth filters with half-power cutoffs at 0.01 and $36 \mathrm{~Hz}$ (roll-off $=12 \mathrm{~dB} /$ oct). Independent component analysis (ICA) was subsequently performed to remove components associated with eye movements and eyeblink activity. The ICA-corrected EEG data were segmented into epochs. Baseline correction was performed by subtracting the mean of the $500-\mathrm{ms}$ precue or prepicture period. Any epochs with EEG voltages exceeding a threshold of $\pm 100 \mu \mathrm{V}$ were excluded from the average.

\footnotetext{
${ }^{2}$ As data from the individual difference measures are not closely related to the hypotheses of this study, they are not shown here.
}

In the regulation task, ICA-corrected EEG data were segmented into epochs that began $500 \mathrm{~ms}$ prior to cue onset and continued for 3,000 ms for SPN analysis. Separate averages were computed for each participant in each of the three cue types (simple viewing cue, detached reappraisal cue, and positive reappraisal cue). Based on previous studies (Moser et al., 2014; Thiruchselvam et al., 2011) and their topographical distribution, SPNs were quantified as average activity collapsed across three frontal electrodes (F1, Fz, F2) over two time windows: early SPN (300-2000 ms) and late SPN (2300$3000 \mathrm{~ms})$. The mean rejection rate of SPN was $9.9 \%(S D=$ $7.5 \%)$, and did not vary as a function of cue type $(p=.99)$. Regarding the LPP, EEG data were segmented into epochs that began $500 \mathrm{~ms}$ prior to the picture onset and continued for 5,000 ms. Separate averages were computed for each participant in each of the four task types (neutral-view, negativeview, detached reappraisal, and positive reappraisal). As the effect of cognitive effort is expected to occur early, previous studies have set the time window of frontal LPP at around 1,000 ms after stimulus onset (Moser et al., 2014; Shafir et al., 2015). Accordingly, and consistent with visual inspection, the early frontal LPPs were quantified as the average activity collapsed across three electrodes (F1, Fz, F2) between $700 \mathrm{~ms}$ and 1,500 ms after picture onset. Based on previous studies (Moser et al., 2014; Paul et al., 2013; Thiruchselvam et al., 2011), central-parietal LPPs were quantified as the average activity collapsed across six electrodes $(\mathrm{CP} 1, \mathrm{CPz}, \mathrm{CP} 2$, $\mathrm{P} 1, \mathrm{Pz}$, and $\mathrm{P} 2$ ) between $300 \mathrm{~ms}$ and $5000 \mathrm{~ms}$ after picture onset. The mean rejection rate of LPP was $12.3 \%(S D=$ $9.6 \%)$, and did not vary as a function of task type $(p=.79)$.

In the re-exposure task, ICA-corrected EEG data were segmented into epochs that began $500 \mathrm{~ms}$ prior to picture onset and continued for $3,000 \mathrm{~ms}$. Separate averages were computed for each participant in each of the four instruction history types (neutral-view history, negative-view history, detached-reappraisal history, and positive-reappraisal history). Based on previous studies (Paul et al., 2013; Thiruchselvam et al., 2011), centralparietal LPPs were quantified as the average activity collapsed across six electrodes $(\mathrm{CP} 1, \mathrm{CPz}, \mathrm{CP} 2, \mathrm{P} 1$, $\mathrm{Pz}$, and $\mathrm{P} 2$ ) during the $300-3,000 \mathrm{~ms}$ time range. Additionally, we conducted an exploratory analysis of frontal LPPs, which were quantified as the average activity collapsed across three electrodes (F1, Fz, F2) during the 700-1,500 ms time range. The mean rejection rate of LPP was $10.9 \%(S D=8.8 \%)$, and did not vary as a function of instruction history type $(p=.66)$. All repeated-measures analysis of variance (ANOVA) results were Greenhouse-Geisser corrected if the sphericity assumption was violated; post-hoc multiple comparisons were performed using Bonferroni-adjusted corrections. Effect sizes are presented as partial eta-squared $\left(\eta_{\mathrm{P}}{ }^{2}\right)$ for $F$ tests and as Cohen's $d$ for $t$ tests. 


\section{Results}

\section{Behavioral data}

\section{Perceived success and effort ratings}

For the perceived success ratings, the main effect of reappraisal type was not significant, $t(24)=1.52, p=.14, d=0.28$. The mean success ratings of the two reappraisal strategies were $\sim 7.69$ on the $1-10$ scale (positive reappraisal: $M=7.50, S D$ $=1.45$; detached reappraisal: $M=7.88, S D=1.26$ ), suggesting that participants successfully complied with reappraisal instructions to implement each reappraisal strategy. Analysis of self-reported effort ratings showed a significant main effect of reappraisal type, $t(24)=5.78, p<.001, d=1.31$, as participants reported expending more effort during positive reappraisal $(M=7.38, S D=1.64)$ than detached reappraisal $(M$ $=4.92, S D=2.10)$ implementation.

\section{Subjective emotional experience}

Regulation task The self-reported valence and arousal ratings were analyzed using a repeated-measures ANOVA, with task type as a within-subjects factor. Regarding valence ratings, a significant main effect of task type was observed, $F(3,72)=$ $63.24, p<.001, \eta_{\mathrm{P}}{ }^{2}=.73$. Pairwise comparisons indicated that negative-view $(M=3.54, S D=0.67)$ was associated with the experience of greater unpleasantness than neutral-view $(M=$ 5.51, $S D=0.50, p<.001, d=-3.33)$. Positive reappraisal $(M$ $=4.57, S D=0.92)$ and detached reappraisal $(M=4.21, S D=$ $0.70)$ were also associated with the experience of greater unpleasantness than was neutral-view ( $p \mathrm{~s}<.001, d \mathrm{~s}<-1.26)$. Both positive $(p<.001, d=1.28)$ and detached $(p<.001, d=$ 0.98 ) reappraisal diminished negative affect relative to negative-view. Positive reappraisal increased pleasantness more than detached reappraisal did ( $p=.04, d=0.44$; see Fig. 1a).

Regarding arousal ratings, a significant main effect of task type was observed, $F(3,72)=32.02, p<.001, \eta_{\mathrm{P}}{ }^{2}=.57$.

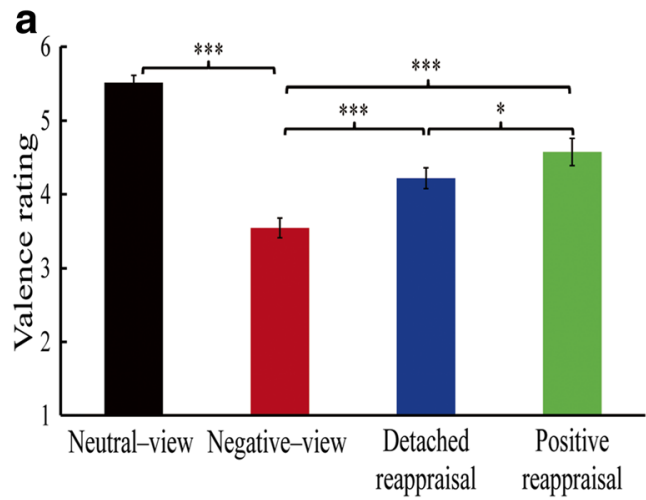

Fig. 1 Mean valence and arousal ratings across four task types in the regulation task. Error bars represent standard errors of the means. * $p<.05$. ** $p<$ $.01 . * * * p<.001$ (Color figure online)



Pairwise comparisons indicated that negative-view $(M=$ $6.00, S D=1.09$ ) elicited greater arousal than neutral-view $(M=4.09, S D=1.48, p<.001, d=1.47)$. Positive reappraisal $(M=5.63, S D=1.07)$ and detached reappraisal $(M=5.22, S D$ $=1.15$ ) elicited greater arousal than did neutral-view $(p s<$ $.001, d \mathrm{~s}>0.85)$. Compared with negative-view, positive reappraisal $(p=.005, d=-0.34)$ and detached reappraisal $(p<$ $.001, d=-0.69$ ) reduced arousal elicited by negative pictures. Detached reappraisal reduced arousal more than positive reappraisal did ( $p=.03, d=-0.37$; see Fig. $1 \mathrm{~b}$ ).

Re-exposure task The self-reported valence and arousal ratings were analyzed using a repeated-measures ANOVA, with instruction history type as a within-subjects factor. Regarding valence ratings, a significant main effect of instruction history type was observed, $F(3,72)=140.11, p<.001, \eta_{\mathrm{P}}{ }^{2}=.85$. Pairwise comparisons indicated that pictures with a negativeview history $(M=3.49, S D=0.76)$ were rated as more unpleasant than those with a neutral-view history $(M=5.31, S D$ $=0.52, p<.001, d=-2.80)$. Pictures with a positive reappraisal history $(M=3.64, S D=0.73)$ or a detached reappraisal history $(M=3.59, S D=0.70)$ were rated as more unpleasant than those with a neutral-view history $(p s<.001, d s<-2.64)$. Pictures with a positive reappraisal history were rated as more pleasant than those with a negative-view history $(p=.02, d=$ 0.20 ). However, pictures with a detached reappraisal history were of a similar degree of pleasantness to those with a negative-view history ( $p=.19, d=0.13$ ). Additionally, there was no significant difference in valence ratings between the reappraisal history types ( $p=1.00, d=0.07$; see Fig. 2a).

Regarding arousal ratings, a significant main effect of instruction history type was observed, $F(3,72)=26.03, p<$ $.001, \eta_{\mathrm{P}}{ }^{2}=.52$. Pairwise comparisons indicated that pictures with a negative-view history $(M=5.46, S D=1.49)$ were rated as more emotionally arousing than those with a neutral-view history $(M=3.87, S D=1.54, p<.001, d=1.05)$. Pictures with a positive reappraisal history $(M=5.26, S D=1.39)$ or a detached reappraisal history $(M=5.39, S D=1.28)$ were rated 

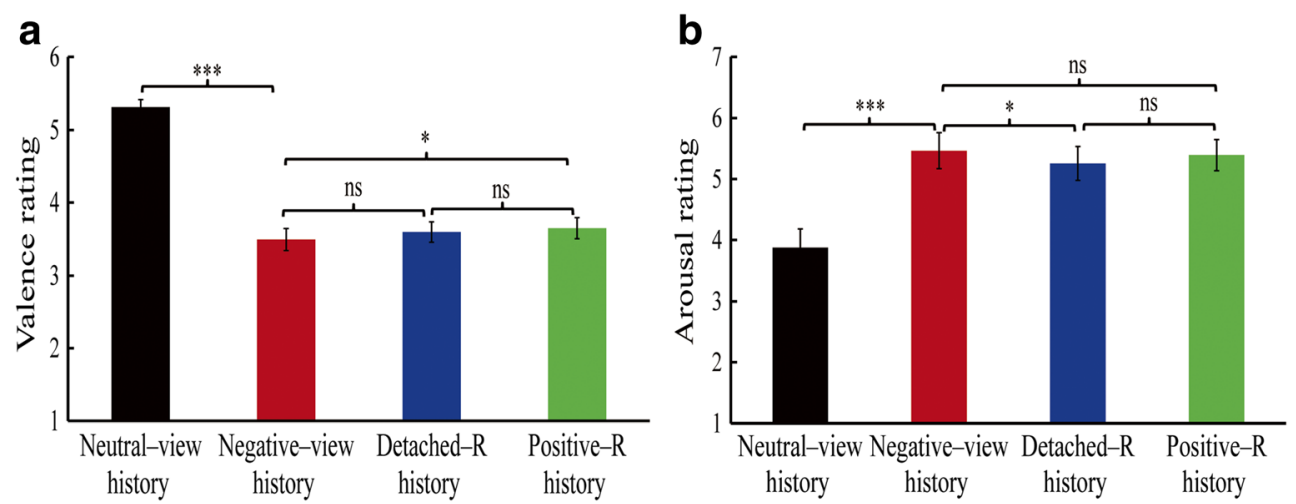

Fig. 2 Mean valence and arousal ratings across four instruction history types in the re-exposure task. Error bars represent standard errors of the means. Detached- $\mathrm{R}=$ detached reappraisal; Positive- $\mathrm{R}=$ positive reappraisal. $* p<.05 . * * * p<.001 . n s>.05$ (Color figure online)

as more emotionally arousing than those with a neutral-view history $(p \mathrm{~s}<.001, d \mathrm{~s}>0.95)$. Pictures with a detached reappraisal history were rated as less emotionally arousing than those with a negative-view history $(p=.03, d=0.14)$. In contrast, pictures with a positive reappraisal history were associated with similar arousal levels to those with a negativeview history $(p=1.00, d=0.05)$. Additionally, there was no significant difference in arousal ratings between the reappraisal history types ( $p=.19, d=0.10$; see Fig. $2 b$ ).

\section{ERP data}

\section{Regulation task}

SPN Figure 3 presents cue-elicited SPN waveforms. A repeated-measures ANOVA examining early SPN amplitudes detected a significant main effect of cue type, $F(2,48)=4.57$, $p=.02, \eta_{\mathrm{P}}^{2}=.16$. Regarding post-hoc pairwise comparisons, detached reappraisal cues $(M=0.70, S D=2.10)$ elicited a larger SPN than positive reappraisal cues $(M=2.13, S D=$ 2.08; $p=.04, d=-0.68)$. The simple viewing cues $(M=$ $1.10, S D=2.25)$ also elicited a larger SPN than positive reappraisal cues ( $p=.058, d=0.49$ ). However, for the late SPN, the main effect of cue type was not significant, $F(2,48)=$ $0.94, p=.40, \eta_{\mathrm{P}}^{2}=.038$.

Frontal LPP Figure 4 presents frontal LPP waveforms elicited by pictures in the regulation task. A repeatedmeasures ANOVA examining frontal LPP amplitudes detected a main effect of task type, $F(3,72)=9.96, p$ $<.001, \eta_{\mathrm{P}}^{2}=.29$. Post-hoc pairwise comparisons indicated that positive reappraisal $(M=5.38, S D=4.52)$ elicited a larger frontal LPP than negative-view $(M=$ 2.58, $S D=4.55 ; p=.005, d=0.62)$, but detached reappraisal did not $(M=2.95, S D=3.84 ; p=1.00, d$ $=0.09)$. Positive reappraisal also elicited a larger frontal LPP than detached reappraisal did $(p=.03, d=0.58)$.
Central-parietal LPP Figure 5 presents central-parietal LPP waveforms elicited by pictures in the regulation task. We first examined whether both reappraisal strategies modulated central-parietal LPPs, and subsequently examined their time course.

Initially, mean central-parietal LPP amplitudes for the entire picture duration (300-5,000 ms) were analyzed using a repeated-measures ANOVA, with task type as a withinsubjects factor. A significant main effect of task type was detected, $F(3,72)=19.16, p<.001, \eta_{\mathrm{P}}^{2}=.44$. In post-hoc pairwise comparisons, negative-view $(M=5.38, S D=3.89)$ elicited a larger LPP than neutral-view $(M=1.16, S D=3.62 ; p$ $<.001, d=1.12)$. Positive reappraisal $(M=3.33, S D=3.25)$ and detached reappraisal $(M=2.05, S D=3.24)$ elicited smaller LPPs than negative-view ( $p$ s $<.01, d$ s $<-0.57)$. Furthermore, detached reappraisal elicited a smaller centralparietal LPP than positive reappraisal $(p=.05, d=-0.39)$.

Subsequently, based on previous studies (Paul et al., 2013; Thiruchselvam et al., 2011), we divided the early time window of central-parietal LPPs (300-1,700 ms) into seven 200$\mathrm{ms}$ time windows (300-500, 500-700, 700-900, 900-1100, $1100-1300,1300-1500$, and $1500-1700 \mathrm{~ms})$. Mean early LPP amplitudes were analyzed using a 4 (task type) $\times 7$ (time window) repeated-measures ANOVA, revealing a significant interaction between task type and time window, $F(18,432)=$ $3.15, p=.003, \eta_{\mathrm{P}}{ }^{2}=.116$. Finally, to determine whether positive and detached reappraisal exhibit differential time courses, we performed planned $t$ tests comparing each reappraisal strategy to negative-view across the seven early LPP time windows. Based on previous studies (Paul et al., 2013; Thiruchselvam et al., 2011), a modified Bonferroni correction (Holm's test) was used to minimize Type I error, ${ }^{3}$ while providing more statistical power than the Bonferroni method.

\footnotetext{
${ }^{3}$ The modified Bonferroni correction for multiple comparisons (Holm's test; Holm, 1979) was performed as follows. The seven $p$ values in each time course analysis of positive or detached reappraisal were ranked from smallest to largest. A unique $\alpha$ level for each $p$ value was subsequently calculated using the following formula: 0.05 / (7- position in sequence +1$)$. Each $p$ value was then compared against its corresponding $\alpha$ value.
} 


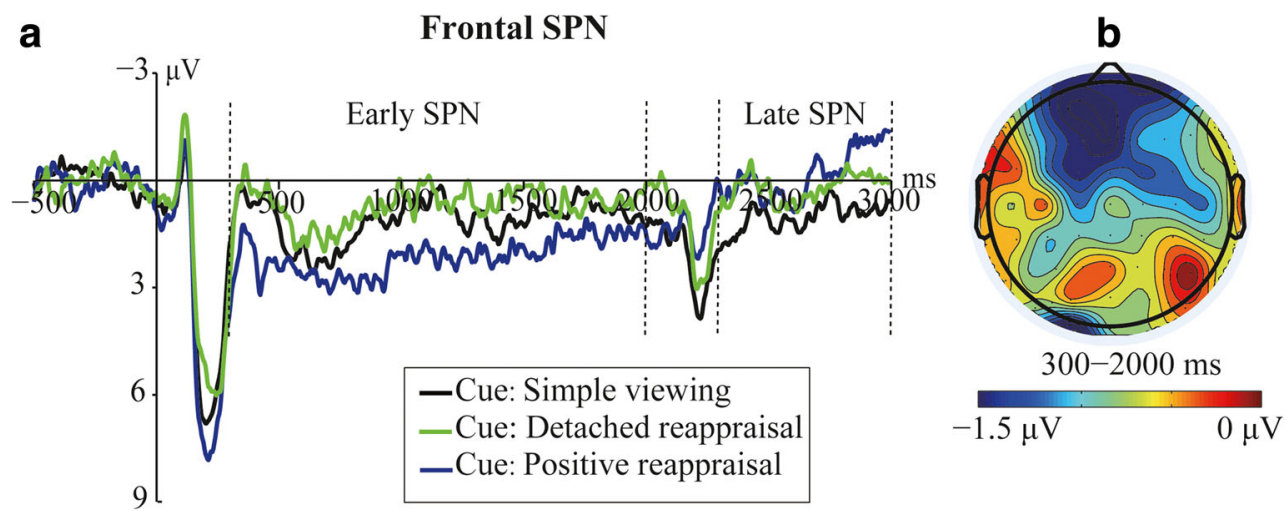

Fig. 3 a Cue-locked SPNs pooled at frontal sites (F1, Fz, F2). b Topographical distribution of the difference wave of detached reappraisal cue minus positive reappraisal cue (Color figure online)

Tables 1 and 2 present the planned comparisons between each reappraisal strategy and negative-view across the seven time windows. Supporting our hypothesis, compared with negative-view, detached reappraisal began to attenuate the central-parietal LPP in the 700-900-ms time window and continued to do so in the subsequent time windows; in contrast, positive reappraisal began to attenuate the central-parietal LPP in the 1,300-1,500-ms time window.

\section{Re-exposure task}

Frontal LPP Figure 6 presents frontal LPP waveforms elicited by pictures in the re-exposure task. A repeated-measures ANOVA examining frontal LPP amplitudes detected a significant main effect of instruction history type, $F(3,72)=23.23$, $p<.001, \eta_{\mathrm{P}}{ }^{2}=.49$. In post-hoc pairwise comparisons, pictures with a negative-view history $(M=6.48, S D=3.30)$ elicited a larger frontal LPP than those with a neutral-view history $(M=$ $1.91, S D=3.90 ; p<.001, d=1.27)$. However, pictures with a positive reappraisal history $(M=6.45, S D=3.66)$ elicited a similar frontal LPP to those either with a negative-view history $(p=1.00, d=0.009)$ or a detached reappraisal history $(M=$ 5.26, $S D=4.06 ; p=.36, d=0.31)$. Furthermore, there was no significant difference in frontal LPP amplitudes between the negative-view history and detached-reappraisal history conditions.

Central-parietal LPP Figure 7 presents central-parietal LPP waveforms elicited by pictures in the re-exposure task. A repeated-measures ANOVA, with instruction history type as a within-subjects factor, was used to determine whether reappraisal history modulated central-parietal LPP amplitudes, revealing a significant main effect of instruction history type, $F(3,72)=25.47, p<.001, \eta_{\mathrm{P}}{ }^{2}=.52$. In post-hoc pairwise comparisons, pictures with a negative-view history $(M=5.30$, $S D=2.88$ ) elicited a larger central-parietal LPP than those with a neutral-view history $(M=1.30, S D=3.42 ; p<.001$, $d=1.26)$. Pictures with a detached reappraisal history $(M=$ $3.71, S D=3.99$ ) elicited a smaller LPP than those with a negative-view history $(p=.006, d=-0.46)$. In contrast, pictures with a positive reappraisal history $(M=4.64, S D=2.80)$ elicited a similar amplitude of LPP to those either with a negative-view history ( $p=.84, d=-0.23$ ) or a detachedreappraisal history ( $p=.65, d=0.27$ ).

Additionally, inspection of the grand averaged waveforms (see Fig. 7) seemed to suggest that LPP for pictures in response to which positive reappraisal was previously instructed was associated with an initial increase in LPP amplitude,
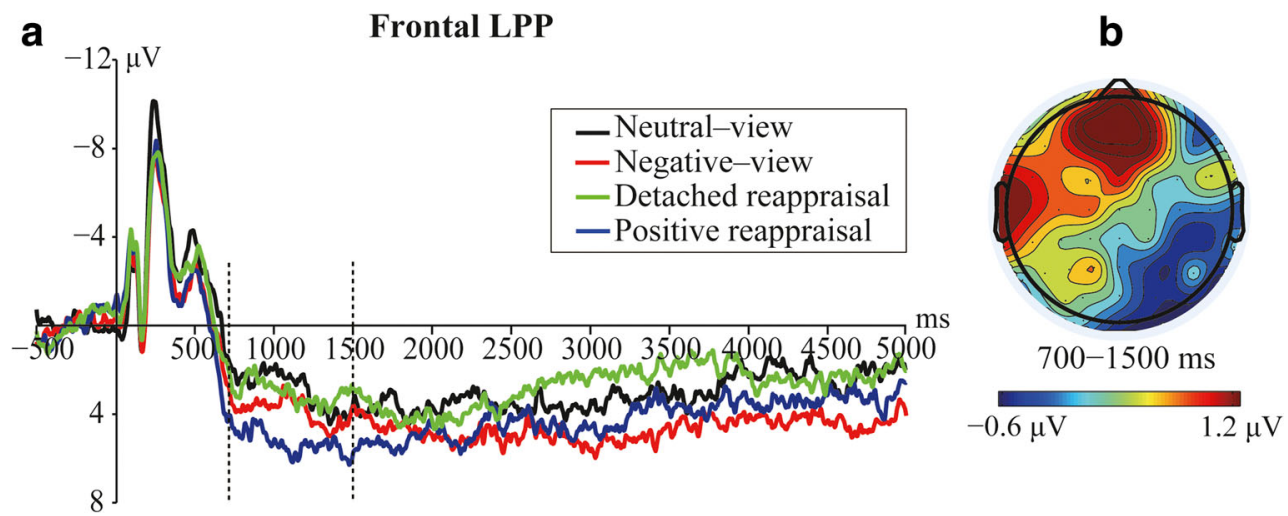

Fig. 4 a Picture-locked LPPs pooled at frontal sites (F1, Fz, F2). b Topographical distribution of the difference wave of positive reappraisal minus detached reappraisal (Color figure online) 


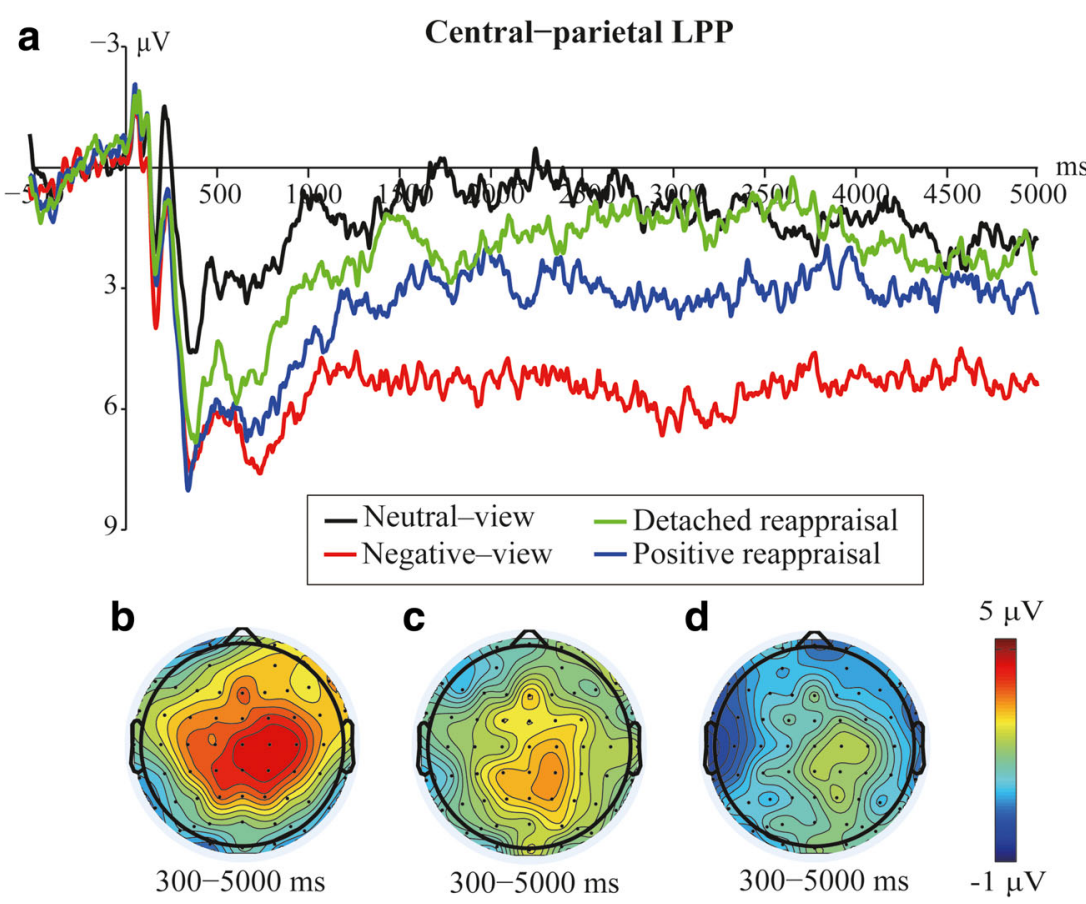

Fig. 5 a Picture-locked LPPs pooled at central-parietal sites $(\mathrm{CP} 1, \mathrm{CPz}$, $\mathrm{CP} 2, \mathrm{P} 1, \mathrm{Pz}, \mathrm{P} 2)$ in the regulation task. Topographical distribution of the difference wave of negative-view minus neutral-view (b), negative-view

while it pointed in the opposite direction later on. A 4 (instruction history type) $\times 7$ (time window) repeated-measures ANOVA examining early LPP amplitudes was used to examine the time course of positive and detached reappraisal's lasting effect, revealing a significant interaction between instruction history type and time window, $F(18,432)=3.45, p=$ $.002, \eta_{\mathrm{P}}^{2}=.126$. Tables $\mathrm{S} 1$ and $\mathrm{S} 2$ present planned comparisons between negative-view history and each reappraisal strategy history across the seven time windows (see Supplementary Materials). Inconsistent with our expectations, the positive reappraisal history was indistinguishable from the negative-view history between 300 and 1,700 ms post-picture onset (Holm's correction); however, the detached reappraisal history began to attenuate the central-parietal LPP in the time window of 900 to $1,100 \mathrm{~ms}$. minus detached reappraisal (c), and negative-view minus positive reappraisal (d) (Color figure online)

\section{Discussion}

To further clarify the differences between detached and positive reappraisal, ERPs were used to compare the temporal dynamics of these strategies during the regulation and reexposure phases. During the regulation phase, detached reappraisal reduced self-reported arousal to a greater degree than positive reappraisal did, whereas positive reappraisal increased pleasantness to a greater degree than detached reappraisal did. Central-parietal LPPs were attenuated under both reappraisal strategies, with the regulation effect of detached reappraisal occurring earlier than positive reappraisal and resulting in a greater attenuation of LPP amplitude. During the re-exposure phase, detached, but not positive, reappraisal history led to a significant attenuation of the central-parietal
Table 1 Means (standard deviations) for pair-wise comparisons between negative-view and detached reappraisal at each 200 ms time window of early centralparietal LPP (300-1,700 ms)

\begin{tabular}{llllll}
\hline Time $(\mathrm{ms})$ & Negative-view & Detached reappraisal & $t$ value & $p$ value & $\begin{array}{l}\text { Holm's } \\
a \text { value }\end{array}$ \\
\hline $300-500$ & $6.24(4.25)$ & $5.39(4.43)$ & 1.65 & .112 & 0.050 \\
$500-700$ & $6.23(4.64)$ & $4.95(3.80)$ & 1.89 & .071 & 0.025 \\
$700-900$ & $6.84(4.44)$ & $5.10(4.02)$ & 3.22 & $.004^{*}$ & 0.016 \\
$900-1100$ & $5.49(4.62)$ & $2.72(3.80)$ & 3.66 & $.001^{*}$ & 0.010 \\
$1100-1300$ & $4.82(4.35)$ & $2.47(3.83)$ & 3.30 & $.003^{*}$ & 0.012 \\
$1300-1500$ & $5.12(4.16)$ & $1.62(3.54)$ & 4.79 & $<.001^{*}$ & 0.007 \\
$1500-1700$ & $5.07(4.72)$ & $1.51(3.56)$ & 4.40 & $<.001^{*}$ & 0.008 \\
\hline
\end{tabular}

* Significance after Holm's correction. Holm's $a$ value was calculated by ordering the seven $p$ values from smallest to largest and then applying the formula: $0.05 /(7-$ position in sequence +1$)$ 
Table 2 Means (standard deviations) for pair-wise comparisons between negative-view and positive reappraisal at each 200-ms time window of early central-parietal LPP (300-1,700 ms)

\begin{tabular}{|c|c|c|c|c|c|}
\hline Time (ms) & Negative-view & Positive reappraisal & $t$ value & $p$ value & $\begin{array}{l}\text { Holm's } \\
a \text { value }\end{array}$ \\
\hline $300-500$ & $6.24(4.25)$ & $6.33(5.49)$ & -0.13 & .895 & 0.050 \\
\hline $500-700$ & $6.23(4.64)$ & $5.91(3.92)$ & 0.51 & .615 & 0.025 \\
\hline $700-900$ & $6.84(4.44)$ & $5.86(4.25)$ & 1.79 & .087 & 0.012 \\
\hline $900-1100$ & $5.49(4.62)$ & $4.41(4.02)$ & 1.77 & .089 & 0.016 \\
\hline $1100-1300$ & $4.82(4.35)$ & $3.51(3.77)$ & 2.33 & .028 & 0.010 \\
\hline $1300-1500$ & $5.12(4.16)$ & $3.30(3.73)$ & 3.47 & $.002 *$ & 0.008 \\
\hline $1500-1700$ & $5.07(4.72)$ & $2.62(3.98)$ & 3.93 & $.001 *$ & 0.007 \\
\hline
\end{tabular}

* Significance after Holm's correction. Holm's $a$-value was calculated by ordering the seven $p$ values from smallest to largest, and then applying the formula: $0.05 /(7-$ position in sequence +1$)$

LPP amplitude. Overall, these results suggest that detached reappraisal and positive reappraisal are characterized by differential temporal dynamics and regulation effects.

Specifically, during the regulation phase, both detached and positive reappraisal effectively modulated individuals' emotional experience, including both valence and arousal. This finding accords with previous studies demonstrating a successful modulation of emotional experience during detached reappraisal implementation (Schönfelder et al., 2014) and the interchangeable implementation of detached and positive reappraisal (Paul et al., 2013; Thiruchselvam et al., 2011). Building on the findings of previous studies (Denny \& Ochsner, 2014; Willroth \& Hilimire, 2016), we found the tested reappraisal strategies differentially modulate the self-reported valence and arousal of negative pictures. Specifically, detached reappraisal reduced arousal more than positive reappraisal did, whereas positive reappraisal increased pleasantness more than detached reappraisal did.
Regarding ERPs, this study's key finding was that detached reappraisal resulted in an earlier and stronger attenuation of central-parietal LPPs than did positive reappraisal. This finding supports the prediction of the conceptual framework of emotion regulation choice (Sheppes et al., 2014), suggesting that the more disengaging detached reappraisal operates earlier and more efficiently than the emotionally engaging positive reappraisal. Most previous studies focused on the differential timing characteristics of various emotion regulation strategies (i.e., distraction, cognitive reappraisal, suppression; Paul et al., 2013; Schönfelder et al., 2014; Thiruchselvam et al., 2011). Our ERP finding adds to the results of these studies, showing that even substrategies of cognitive reappraisal possess differential timing characteristics. Accordingly, emotion regulation models should consider the point in time at which a given reappraisal strategy is to be implemented.

The SPN and frontal LPP also provide valuable insights into the separability of detached and positive reappraisal and their

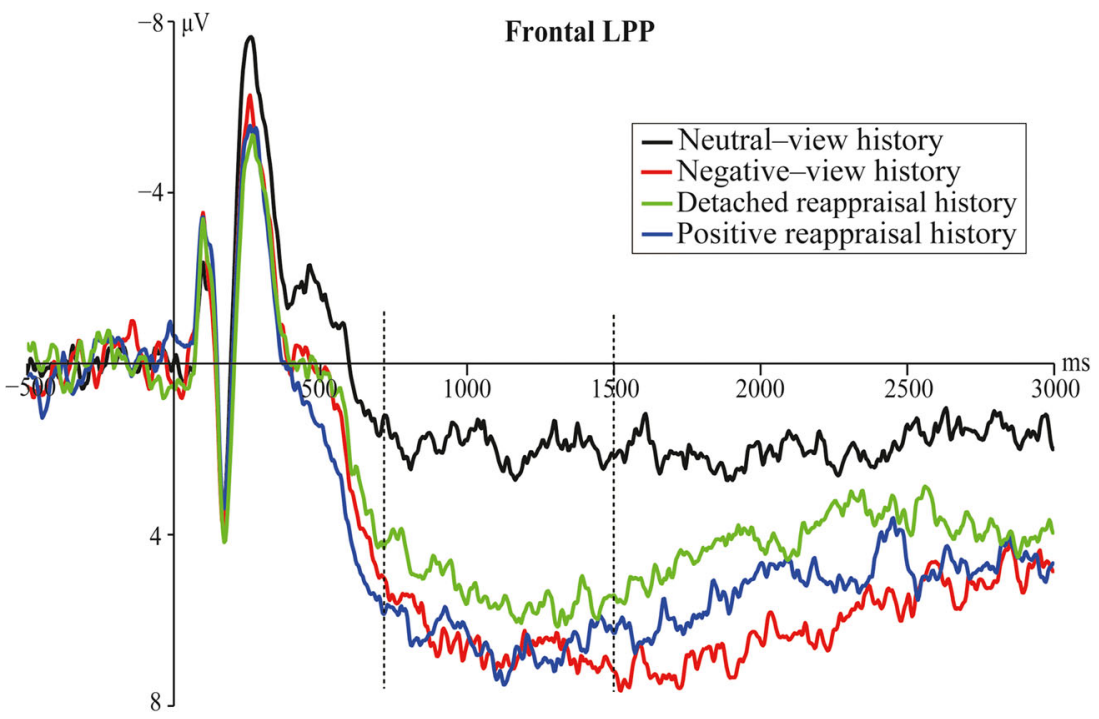

Fig. 6 Picture-locked LPPs by instruction history pooled at frontal sites (F1, Fz, F2) in the re-exposure task (Color figure online) 


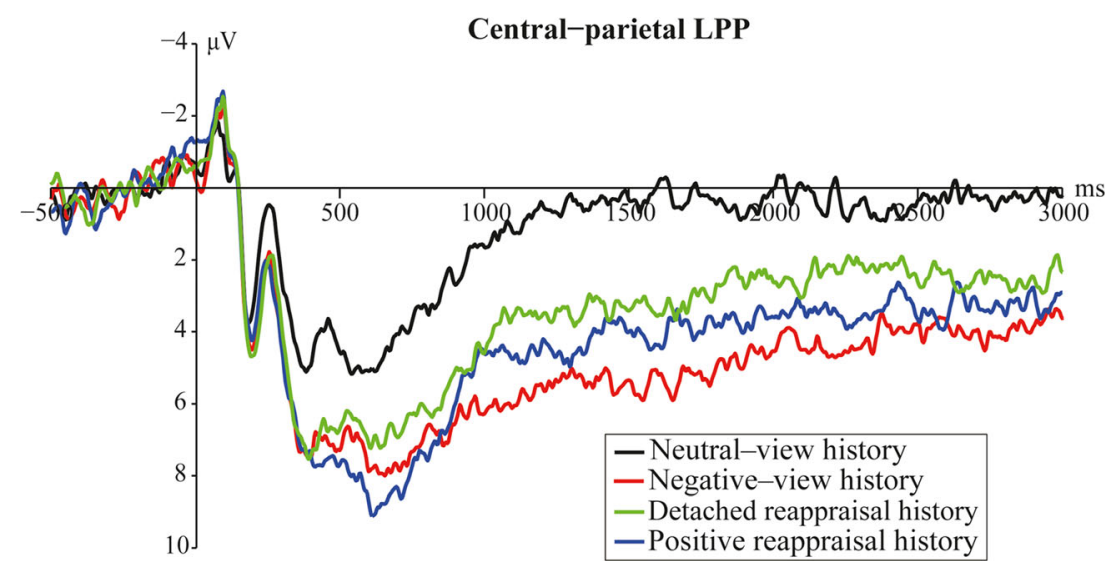

Fig. 7 Picture-locked LPPs by instruction history pooled at central-parietal sites (CP1, CPz, CP2, P1, Pz, P2) in the re-exposure task (Color figure online)

underlying regulation processes. During the anticipatory period, detached reappraisal cues elicited a greater frontal SPN than positive reappraisal cues did. SPN reflects anticipatory processes in preparation for the impending stimulus (Brunia et al., 2012; Moser et al., 2014; Seidel et al., 2015); therefore, our finding suggests that individuals prepare more before engaging in detached reappraisal than in positive appraisal. Additionally, in our experimental design, the simple viewing cue did not indicate the valance of the upcoming picture; this may have elicited some uncertainty in participants regarding the stimulus' affective valence. In contrast, no such uncertainty exists in the positive reappraisal condition, as participants responded only to negative stimuli. Research has found that uncertain cues elicit more pronounced SPN than certain cues do during the early anticipation phase (Seidel et al., 2015). Therefore, the finding of increased SPN after simple viewing cues relative to positive reappraisal cues may be explained by increased uncertainty regarding the upcoming picture valence during simple viewing. Nevertheless, this interpretation should be treated cautiously, as evidence showing that uncertain cues produce reduced SPN relative to certain cues is also emerging (Lin et al., 2014).

Consistent with Moser et al. (2014), positive reappraisal elicited a larger frontal LPP than simple viewing of negative pictures did during the regulation period; however, this enhancement of frontal LPP was not observed during detached reappraisal. These findings indicate that enhanced frontal LPP is associated with positive reappraisal specifically, rather than reappraisal in general. The frontal LPP is linked to cognitive effort or attentional control (Moser et al., 2014; Shafir et al., 2015); hence, our finding of increased frontal LPP under positive reappraisal suggests that implementing this strategy requires greater cognitive effort or attentional control than implementing detached reappraisal. Supporting this interpretation, participants reported that performing positive reappraisal was more effortful. We therefore speculate that an initial recruitment of increased cognitive effort or attentional control is required for the successful implementation of positive reappraisal, which might delay the occurrence of a modulating effect of positive reappraisal on the centralparietal LPP.

With respect to the re-exposure phase, our findings agree with those of previous studies (Macnamara et al., 2011; Thiruchselvam et al., 2011), indicating that reappraisal is able to exert lasting effects on re-encountered stimuli. Extending the findings of these studies, we found that detached reappraisal enduringly affected self-reported arousal and centralparietal LPPs, whereas positive reappraisal only enduringly affected pleasantness. We speculate that the different lasting effects of detached and positive reappraisal are mediated by beneficial (i.e., less arousing/more pleasant) memory encoding in the regulation phase. During regulation, detached reappraisal modulated arousal more so than pleasantness, while positive reappraisal showed the converse. With time, the reappraisal's regulatory effects are likely to weaken. Consequently, detached reappraisal led to less arousing representations of negative pictures in memory, thereby rendering them less arousing upon re-exposure; however, positive reappraisal largely resulted in more pleasant representations of negative pictures, thereby rendering them more pleasant upon re-exposure. The central-parietal LPP is linked to the arousal levels of emotional pictures (Cuthbert et al., 2000; Hajcak \& Nieuwenhuis, 2006; Paul et al., 2013), and positive reappraisal failed to exert a lasting effect on self-reported arousal; therefore, LPP is likely to be similar between positive reappraisal history and negative-view history. Nonetheless, the observed lasting effects might also be interpreted in terms of reimplementing previously used regulation strategies. The interval between the regulation task and the re-exposure task was relatively short; therefore, it is plausible that a given picture easily elicits the regulation strategy previously applied to it. This explanation seems unlikely, as participants apparently did not reimplement the effortful strategy (positive reappraisal) during the re-exposure phase, as indicated by positive reappraisal's lack of lasting effect on frontal LPPs. 
The different lasting effects of detached and positive reappraisal have a profound implication for these strategies' relative long-term utility. Previous studies suggest some strategies may exacerbate distress in the long term (Sheppes et al., 2014; Thiruchselvam et al., 2011). For example, distraction, compared with simple viewing, comes at a long-term cost by eliciting stronger emotional responses to reencountered negative stimuli (Thiruchselvam et al., 2011). Our findings indicate that detached and positive reappraisal strategies are preferable in this regard, and that each of these strategies confers a different type of long-term benefit: detached reappraisal attenuates arousal; positive reappraisal increases perceived pleasantness. Such different benefits therefore suggest that the particular reappraisal strategy adopted ought to be a function of the individual's emotion regulation goals.

This study has some limitations that offer direction to future research. First, according to the typical conception of positive reappraisal, an effective implementation of positive reappraisal should shift participants' emotional experience into positive affect; however, the pleasantness ratings in the positive reappraisal condition remained below those in the neutral-view condition. Positive reappraisal's efficacy can increase with practice (Denny \& Ochsner, 2014); therefore, future research should conduct positive reappraisal training to improve its effectiveness. Second, we used the simple viewing cue for presenting neutral and negative pictures. This might introduce some uncertainty among participants regarding the valence of the picture they will be exposed to. Consequently, our results do not permit the inference of whether both types of reappraisal cue elicit greater preparation than negative-view cues do. Therefore, future research should use different cues in simple viewing of neutral and negative pictures to control for the potential effect of uncertainty. Third, our classification of early and late frontal SPN time windows should be interpreted with caution. It is hard to interpret the distinction between early and late frontal-SPN as a cue was presented during the "early" window, whereas a blank screen and a fixation cross were presented during the "late" window. Perhaps the appearance of the fixation cross in the late time window activated the same preparation for the upcoming pictures. Consequently, we did not observe a significant effect of cue type on the late SPN. Thus, to properly examine early and late SPNs, instruction cues should be continuously presented until the following pictures appear.

To summarize, we have first demonstrated that detached and positive reappraisal strategies exhibit differential temporal dynamics. Further, our findings reveal the potential benefits of detached reappraisal for arousal modulation, and positive reappraisal for valence modulation in the short- or long-term. Thus, emotion regulation models should regard cognitive reappraisal as a heterogeneous strategy, addressing not only the differential regulation effects of detached and positive reappraisal on arousal and valence but also the point in time when these two reappraisal strategies are implemented.

\section{Compliance with ethical standards}

Funding This work was supported by grants from the National Natural Science Foundation of China (Grant Nos. NSFC31470977 and NSFC31600919), the National Social Science Foundation of China (Grant No. 15BSH084), the Social Science and Humanity on Young Fund of the Ministry of Education (Grant No. 15YJC190016), the China Postdoctoral Science Foundation (Grant Nos. 2015M572517 and 2016T90882), the Natural Science Basic Research Plan in Shaanxi Province of China (Grant No. 2016JQ3019), the Program of Key Science and Technology Innovation Team of Shaanxi Province (Grant No. 2014KTC-18), the Fundamental Research Funds for the Central Universities (Grant No. Senqing Qi 2017), the Interdisciplinary Incubation Project of Learning Science of Shaanxi Normal University (Grant No. SYSK201510), and the Shaanxi Postdoctoral Science Foundation.

\section{References}

Brunia, C. H., van Boxtel, G. J., \& Böcker, K. B. (2012). Negative slow waves as indices of anticipation: The Bereitschaftspotential, the contingent negative variation, and the stimulus-preceding negativity. In S. J. Luck \& E. S. Kappenman (Eds.), Oxford handbook of ERP components (pp. 189-207). New York, NY: Oxford University Press.

Buhle, J. T., Silvers, J. A., Wager, T. D., Lopez, R., Onyemekwu, C., Kober, H., ... Ochsner, K. N. (2014). Cognitive reappraisal of emotion: A meta-analysis of human neuroimaging studies. Cerebral Cortex, 24, 2981-2990.

Cuthbert, B. N., Schupp, H. T., Bradley, M. M., Birbaumer, N., \& Lang, P. J. (2000). Brain potentials in affective picture processing: Covariation with autonomic arousal and affective report. Biological Psychology, 52, 95-111.

Delorme, A., \& Makeig, S. (2004). EEGLAB: An open source toolbox for analysis of single-trial EEG dynamics including independent component analysis. Journal of Neuroscience Methods, 134, 9-21.

Denny, B. T., \& Ochsner, K. N. (2014). Behavioral effects of longitudinal training in cognitive reappraisal. Emotion, 14, 425-433.

Dorfel, D., Lamke, J. P., Hummel, F., Wagner, U., Erk, S., \& Walter, H. (2014). Common and differential neural networks of emotion regulation by detachment, reinterpretation, distraction, and expressive suppression: A comparative fMRI investigation. NeuroImage, 101, 298-309.

Gross, J. J. (2014). Emotion regulation: Conceptual and empirical foundation. In J. J. Gross (Ed.), Handbook of emotion regulation (2nd ed., pp. 3-20). New York, NY: Guilford Press.

Hajcak, G., Dunning, J. P., Foti, D., \& Weinberg, A. (2014). Temporal dynamics of emotion regulation. In J. J. Gross (Ed.), Handbook of emotion regulation (2nd ed., pp. 441-474). New York, NY: Guilford Press.

Hajcak, G., MacNamara, A., \& Olvet, D. M. (2010). Event-related potentials, emotion, and emotion regulation: An integrative review. Developmental Neuropsychology, 35, 129-155.

Hajcak, G., \& Nieuwenhuis, S. (2006). Reappraisal modulates the electrocortical response to unpleasant pictures. Cognitive, Affective, \& Behavioral Neuroscience, 6, 291-297.

Holm, S. (1979). A simple sequentially rejective multiple test procedure. Scandinavian Journal of Statistics, 6, 65-70 
Huang, Y. X., \& Luo, Y. J. (2004). Native assessment of international affective picture system. Chinese Mental Health Journal, 9, 631-634.

Lang, P. J., Bradley, M. M., \& Cuthbert, B. N. (2008). International affective picture system (IAPS): Affective ratings of pictures and instruction manual (Technical Report A-8). Gainesville: University of Florida.

Lin, H. Y., Gao, H. W., You, J., Liang, J. F., Ma, J. P., Yang, N., ... Jin, H. (2014). Larger N2 and smaller early contingent negative variation during the processing of uncertainty about future emotional events. International Journal of Psychophysiology, 94(3), 292-297.

Lopez-Calderon, J., \& Luck, S. J. (2014). ERPLAB: An open-source toolbox for the analysis of event-related potentials. Frontiers in Human Neuroscience, 8, 213.

Macnamara, A., Ochsner, K. N., \& Hajcak, G. (2011). Previously reappraised: The lasting effect of description type on pictureelicited electrocortical activity. Social Cognitive and Affective Neuroscience, 6, 348-358.

McRae, K., Ciesielski, B., \& Gross, J. J. (2012). Unpacking cognitive reappraisal: Goals, tactics, and outcomes. Emotion, 12, 250-255.

Moser, J. S., Hartwig, R., Moran, T. P., Jendrusina, A. A., \& Kross, E. (2014). Neural markers of positive reappraisal and their associations with trait reappraisal and worry. Journal of Abnormal Psychology, 123, 91-105.

Moser, J. S., Krompinger, J. W., Dietz, J., \& Simons, R. F. (2009). Electrophysiological correlates of decreasing and increasing emotional responses to unpleasant pictures. Psychophysiology, 46, 17-27.

Ochsner, K. N., Ray, R. D., Cooper, J. C., Robertson, E. R., Chopra, S., Gabrieli, J. D., \& Gross, J. J. (2004). For better or for worse: Neural systems supporting the cognitive down-and up-regulation of negative emotion. Neurolmage, 23, 483-499.
Paul, S., Simon, D., Kniesche, R., Kathmann, N., \& Endrass, T. (2013). Timing effects of antecedent- and response-focused emotion regulation strategies. Biological Psychology, 94, 136-142.

Schönfelder, S., Kanske, P., Heissler, J., \& Wessa, M. (2014). Time course of emotion-related responding during distraction and reappraisal. Social Cognitive and Affective Neuroscience, 9, 1310-1319.

Seidel, E. M., Pfabigan, D. M., Hahn, A., Sladky, R., Grahl, A., Paul, K., ... Lamm, C. (2015). Uncertainty during pain anticipation: The adaptive value of preparatory processes. Human Brain Mapping, 36, 744-755.

Shafir, R., Schwartz, N., Blechert, J., \& Sheppes, G. (2015). Emotional intensity influences pre-implementation and implementation of distraction and reappraisal. Social Cognitive and Affective Neuroscience, 10, 1329-1337.

Sheppes, G., Scheibe, S., Suri, G., Radu, P., Blechert, J., \& Gross, J. J. (2014). Emotion regulation choice: A conceptual framework and supporting evidence. Journal of Experimental Psychology: General, 143, 163-181.

Shiota, M. N., \& Levenson, R. W. (2012). Turn down the volume or change the channel? Emotional effects of detached versus positive reappraisal. Journal of Personality and Social Psychology, 103, 416-429.

Thiruchselvam, R., Blechert, J., Sheppes, G., Rydstrom, A., \& Gross, J. J. (2011). The temporal dynamics of emotion regulation: An EEG study of distraction and reappraisal. Biological Psychology, 87, 84-92.

Willroth, E. C., \& Hilimire, M. R. (2016). Differential effects of self- and situation-focused reappraisal. Emotion, 16, 468-474. 